\title{
Monitoring drug-induced changes in lysosomal number and volume in live cells using a novel red fluorescent molecular probe
}

\author{
J Coleman, Y Xiang, P Pande, D Shen, D Gattica \& W Patton \\ R\&D, Enzo Life Sciences, Farmingdale, NY 11735
}

Lysosomes are membrane-bound sub-cellular organelles involved in the degradation of macromolecules and pathogens in diverse processes including endocytosis, phagocytosis and autophagy. Lysosomal morphology varies with the state of the cell and its degree of degradative activity, and the vesicles can have pieces of membranes, vacuoles, granules and even parts of mitochondia within them. They are typically spherical vesicles, ranging in size from 0.2 to 2 micrometers in diameter. The lumen of lysosomes and other acidic organelles is characterized by low $\mathrm{pH}$, generated via proton-pumping vacuolar ATPases.

Conventional fluorescent probes for highlighting lysosomal compartments fail to be retained within the organelles once vesicular $\mathrm{pH}$ values begin to rise due to concentration of certain drugs within the vacuolar lumen. We speculated that such probes would accumulate to differing extents based upon differences in the ionization constants of the different substituent groups and the overall membrane lipid permeability of the probes. We describe a novel red-fluorescent probe that was specifically developed to selectively sequester in acidic organelles by a mechanism that likely involves protonation and retention within the membranes of the organelles. However, by careful selection of titratable groups on the probe, staining has been extended into the vacuoles of cells pretreated with weakly basic, cell-permeant compounds, such as the anti-malarial drug chloroquine.

Chloroquine induces vacuolation in mammalian cells, as a result of osmotic swelling and expansion of the lysosomal compartment, arising from the accumulation of the drug molecules in that organelle [1]. HeLa human cervical carcinoma cells and U2OS human bone osteosarcoma epithelial cells treated with $300 \mu \mathrm{M}$ chloroquine for 4 hours show a dramatic increase in lysosome-like vesicle number and volume, confirming that the probe is associated with this subcellular compartment. This allows the probe to be employed for highlighting lysosome-like organelles under certain conditions, such as lysomotrophic drug treatment, wherein cells produce lysosome-like bodies that contain most of the degradative enzymes of the lysosome, but are not as acidic as the parent organelle. Using quantitative imaging, the half maximal effective concentration $\left(\mathrm{EC}_{50}\right)$ of chloroquine was estimated.

The newly developed red fluorescent probe was found to be especially suitable for fluorescence co-localization imaging with Green Fluorescent Protein (GFP)-tagged fusion proteins, providing spectrally pure red signal with minimal spectral spill over into the fluorescein emission channel. By contrast, many red-emitting lysosomal probes photo-convert to green fluorescent species or display metachromatic artifacts wherein emission occurs both in the red and green regions of the spectrum, leading to spurious 
results in GFP co-localization experiments $[2,3]$. The novel probe is highly resistant to photo-bleaching, concentration quenching and photo-conversion, making it particularly suitable for microscopic imaging applications. We demonstrate multiplexing of the probe with Hoechst 33342, a cell-permeable DNA intercalating dye and GFP fusion proteins for the determination of the subcellular localization of a targeted protein. Specifically, nuclei, lysosomes and mitochondria were simultaneously imaged in cells, the latter using a GFP fusion of cytochrome c.

In conclusion, the described red fluorescent probe is suitable for highlighting lysosomes and lysosome-like vesicles and is fully compatible with various fluorescent proteins and fluorescent dyes in multi-color imaging and detection studies. One potential application of the new fluorescent probe is in preclinical drug safety assessment (ADME-Tox) using in vitro cell culture models to aid in the drug development process. Such a high-content screening (HCS) approach could serve to better model human drug responses, while simultaneously conducting safety assessments more quickly, more thoroughly and more cost effectively. Analogous assays might find application in the identification of chemical or biological threat agents by rapid assessment of compounds leading to cellular stress.

\section{References:}

[1] A. Michihara et al. Biol. Pharm. Bull. 28 (2005) 947

[2]E. Freundt et al. Cell Res 17 (2007) 956

[3] F. Nadrigny et al Biophys J. 93 (2007) 969 